\title{
Potentials of locally manufactured wound-field flux switching wind generator in South Africa
}

\author{
Udochukwu B. Akuru ${ }^{1,2^{*}}$, Maarten J. Kamper ${ }^{1}$ \\ 1. Department of Electrical and Electronic Engineering, Stellenbosch University, Stellenbosch, 7600, South Africa \\ 2. Department of Electrical Engineering, University of Nigeria, Nsukka, 410001, Enugu State, Nigeria

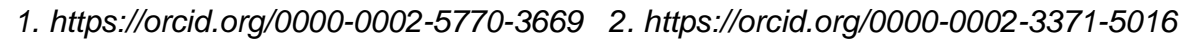

\begin{abstract}
The China-based monopoly of high-energy permanent magnet materials used in modern wind generators impact the economic viability and local content value of most wind turbines installed in South Africa, especially large installations. It is possible to design with less expensive excitation technologies using locallysourced wound-field electromagnets, which might promote local content. This study involves the optimum design performance comparison of the wound-field flux switching machine (WF-FSM) technology based on two variants - Design I and II (D-I and D-II) - the difference being in the arrangement of their DC woundfield coils. The machines are evaluated using finite element analyses (FEA) with optimum performance emphasised on design parameters such as torque density, efficiency and power factor. The selected design targets are meant to improve the performance to cost fidelity of the proposed wind generator variants. In $2 D$ FEA, D-II can produce up to $18.8 \%$ higher torque density $\left(\mathrm{kNm} / \mathrm{m}^{3}\right)$ and $17.1 \%$ lesser loss per active volume $\left(\mathrm{kW} / \mathrm{m}^{3}\right)$ than D-I. In 3D FEA, the torque density of D-II remains higher at $10.6 \%$, but its loss per active volume increases by $15 \%$ compared to D-I. The discrepancy observed in $2 D$ and $3 D$ FEA is due to an underestimation of the end-winding effects in D-II. The power factor of D-II is higher than D-I, both in $2 D$ and $3 D$ FEA, which may translate to lower kVA ratings and inverter costs. A higher total active mass ensues for the studied WF-FSMs than a conventional direct-drive PMSG, but avoiding rare earth PMs translate to significantly lower costs.
\end{abstract}

Keywords: finite element analyses; technology localisation, prototype, South Africa, rare earth-free, wind power industry

\section{Highlights}

- Two WF-FSM variants - D-I and D-II - are optimally compared in FEA for wind generator applications at medium-scale power level.

- $\quad$-I displays better loss per active mass while D-II displays better torque density and power factor.

- Both WF-FSM variants yield heavier but relatively cheaper wind generators when compared to a conventional PMSG.

Journal of Energy in Southern Africa 30(2): 110-117

DOI: https://dx.doi.org/10.17159/2413-3051/2019/v30i2a6315

Published by the Energy Research Centre, University of Cape Town ISSN: 2413-3051

This work is licensed under a Creative Commons Attribution-ShareAlike 4.0 International Licence

https://journals.assaf.org.za/jesa

Sponsored by the Department of Science and Technology

Corresponding author: Tel: +27 718165427;

email: udochukwu.akuru@unn.edu.ng 


\section{Introduction}

The latest global status report on renewable energy indicates that wind power led global renewable capacity for the third year in a row, with a cumulative installed total of $539 \mathrm{GW}-24.5 \%$ of the world total [1]. The same report shows that South Africa ranked among the top counties in Africa for cumulative non-hydropower renewable energy capacity as at the end of 2017. Furthermore, it remains the only African country to have commissioned wind power projects in 2017 [1], while also being the topmost in Africa for installed wind power capacity at 1980 MW, along with an estimated cumulative capacity of 11442 MW by 2030 [2]. To meet its planned renewable energy targets, South Africa has benchmarked, among other things, affordable electricity and technology localisation as key objectives of its draft Integrated Resource Plan 2018 [2].

A key component of the wind turbine architecture is the wind generator. It is commonly taken that doubly-fed induction generators (DFIGs) are the workhorse wind generators in the wind power industry [3]. But with the advent of larger direct-drive wind turbines, permanent magnet synchronous generators (PMSGs) are becoming increasingly relevant due to their propensity for improved torque density designs and operational efficiency performance $[4$, 5]. However, with the growing popularity of PMSGs comes the fact that the high cost of rare earth PMs facilitated by a monopolised market structure, with recent impulsive price swings, is one reason for recent alternative non-PM solutions, so-called rare earth-free wind generator technologies [5].

The PM flux switching machine (PM-FSM) has been commercialised for $3 \mathrm{MW}$ wind turbines [6]. FSM is a re-emerging class of double-salient machines with stator-mounted and robust rotor features which makes it suitable for certain drivetrain applications [7, 8]. Just like synchronous machines, FSMs can be excited with PM (e.g., PM-FSMs) or DC wound-field coils such as wound-field FSMs (WFFSMs). Importantly, the design and operational characteristics of the WF-FSM topology is unlike its conventional wound-rotor synchronous machine (WRSM) counterpart, such that it does not require slip rings and brushes for its DC field current excitation. To this end, the reliability of WF-FSMs is improved.

In South Africa, manufacturing and installation of wind turbines translates to premium on the imported raw materials or technology, at least, of the rare earth PMs mostly deployed in such designs. Based on estimations, $23 \%$ of global installed wind turbines use PMSGs which are designed with rare earth elements [5]. Therefore, for large wind turbines, the PM content mass, implying cost, will increase as the power level increases, especially for direct-drive wind generator systems as illustrated in Figure $1[5,9]$. To this end, there is the possibility of designing wind generators with cheaper excitation technologies such as wound-field (WF) electromagnets which can be locally produced, and in this way the localisation drive of wind power infrastructure could be enhanced in the long run. While a $300 \mathrm{~kW}$ direct-drive PMSG has been fully developed and presented for the South African wind power industry [10], none which uses non-PM technology has been reported.

This study involves the semi-optimised design of the FSM technology based on two proof-of-concept wound-field variants, shown in Figure 2 as Designs I and II (D-I and D-II for short), at $300 \mathrm{~kW}$ power levels. The prescribed power level of the proposed wound-field machines is the so-called medium-scale power range [9]. In Figure 2, the DC coils of D-I overlaps transversally across the adjacent armature coils, while that of D-II displays a parallel overlap winding array between the DC and armature coils.

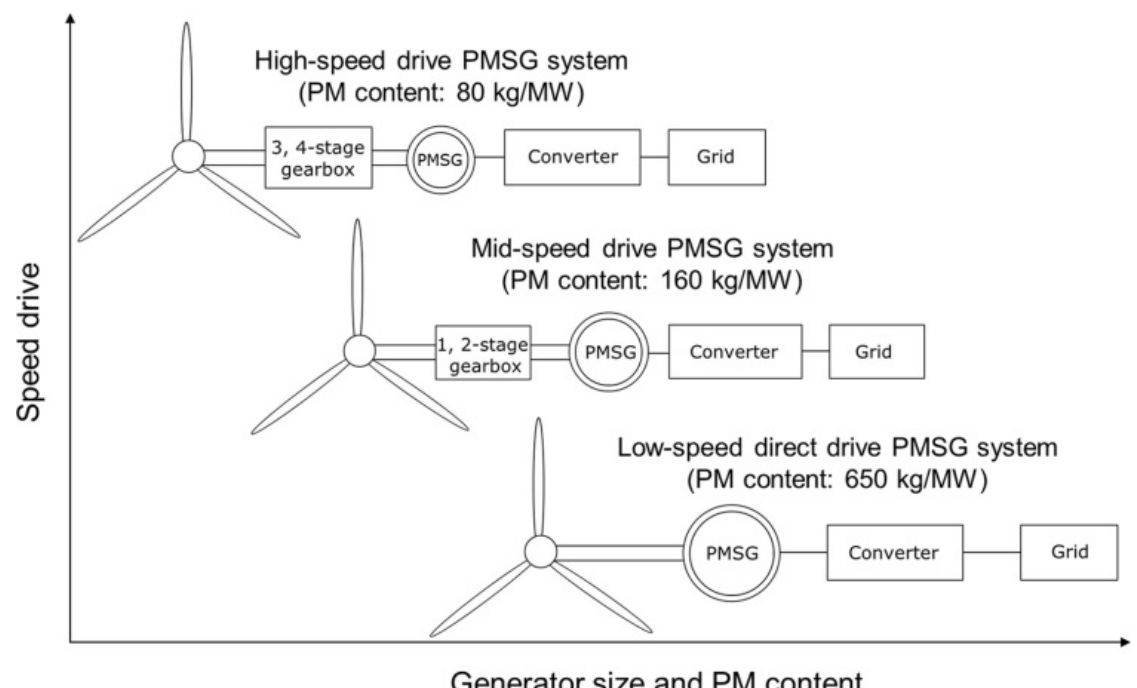

Figure 1: Wind turbines designed with PMSGs [5]. 


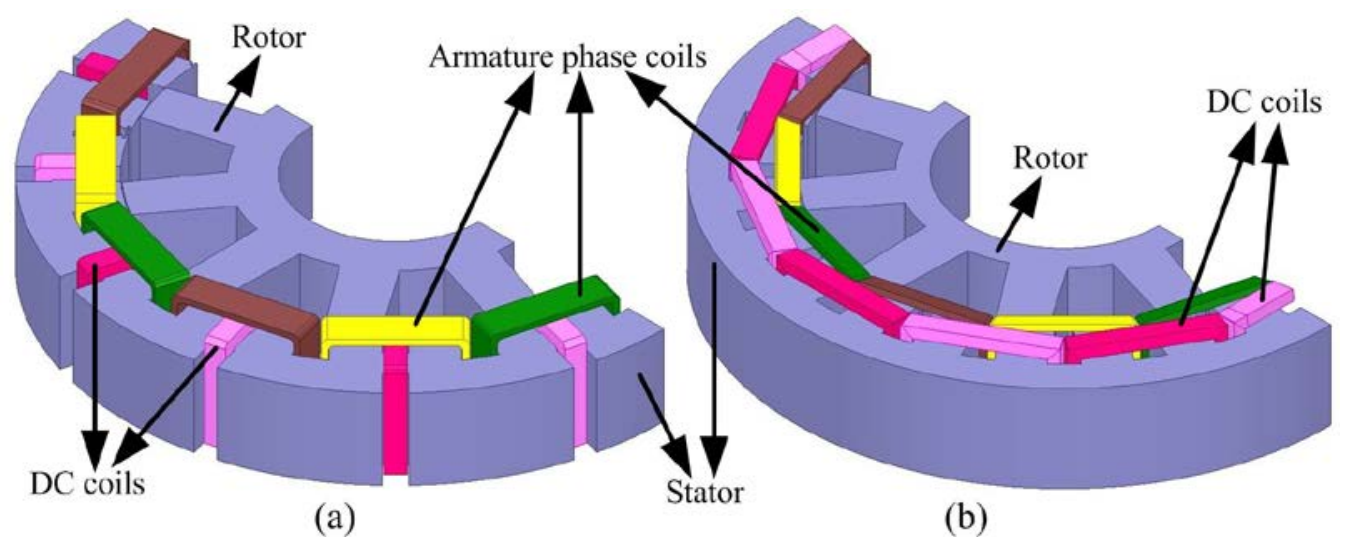

Figure 2: Presentation of the selected WF-FSM designs: (a) D-I, and (b) D-II.

An exhaustive characterisation of the design concept shown as D-I has been undertaken by the authors for both rare earth and rare earth-free wind generator applications, at different power levels [11]. Although D-II is a well-established WF-FSM design concept, there is no clear consideration of it as wind generator designs, not to mention the authors' ignorance of any comparison existing between the designs. Over and above, the WF-FSM generator concept is nominated for this study because, unlike the WRSM, it possesses brushless DC excitation scheme and robust rotor topology.

The proposed machines are primarily evaluated using finite element analyses (FEA) with optimum performance emphasised on design parameters such as torque density, efficiency and power factor, among others. The study is patterned similarly to that performed by Potgieter and Kamper [9], and so makes no promises on the mechanical and thermal fidelity of the design process. The selected design targets are meant to improve the electromagnetic performance to cost index of the proposed generator variants for a medium-speed wind energy drive system as shown in Figure 1. At the end of the study, an existing $10 \mathrm{~kW}$ manufactured prototype of D-I is experimentally reported to validate the results.

Meanwhile, a possible limitation may occur in the initial results of the studied machines at $300 \mathrm{~kW}$ because of the end-winding formulation assumed in the 2D FEA procedure. Besides, because of the 2D nature of the initial static FEA results, the level of magnetic saturation cannot be ascertained, but in $3 \mathrm{D}$ transient FEA. Thus, any such doubts initially accruing from the 2D FEA solutions have been clarified based on the 3D transient FEA calculations, also undertaken in this study.

\section{Optimisation process}

As already mentioned, two WF-FSM variants are benchmarked and initially designed to achieve the reference generated output power $\left(P_{g}\right)$ at $300 \mathrm{~kW}$. The machines are designed with a robust static FEA tool developed in-house, the so-called SEMFEM package [12], which speeds up the simulation time while maintaining reasonable accuracy on the solutions. After obtaining the reference design, the optimisation design is set up and interfaced to the FEA models using a commercial optimiser (VisualDoC [13]), as shown in Figure 3. The optimisation is based on a simple gradient approach called the modified method of feasible directions (MMFD). Further information about the MMFD approach can be found in [14]. The optimisation problem is then processed as follows:

Minimise: $M_{T o t}$

Subject to: $P_{g} \approx 300 \mathrm{~kW}$

$$
\begin{aligned}
& P F \geq 0.8 \\
& \eta \geq 95 \% \\
& \Delta T_{L} \leq 10 \%
\end{aligned}
$$

where $\left(M_{T o t}\right)$ is the total active mass, $(P F)$ is power factor, $(\eta)$ is efficiency and $\left(\Delta T_{L}\right)$ is the peak-to-peak torque ripple at rated load.

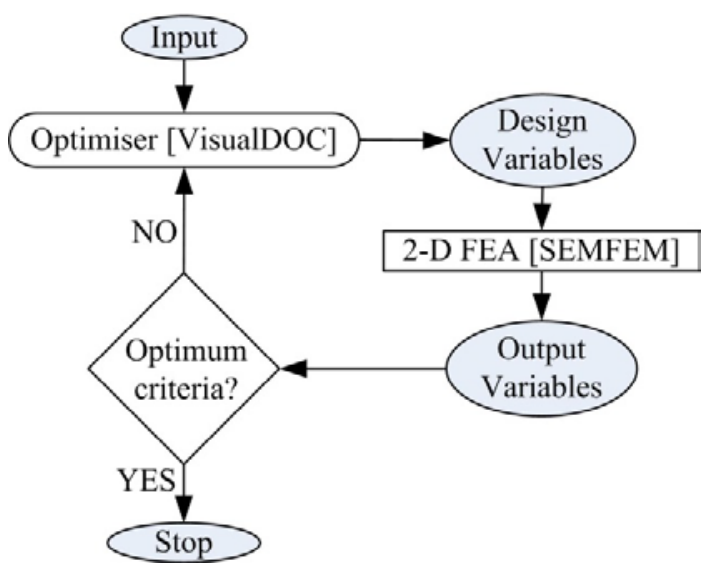

Figure 3: An illustration of the design optimisation procedure. 
A total of 14 design parameters were nominated for D-I, and 13 for D-II. In each case, the design parameters include both dimensional and non-dimensional variables, such as stator outer diameter $\left(D_{o}\right)$, stator inner diameter $\left(D_{i}\right)$, stack length $(l)$, armature current density $\left(J_{s}\right)$ and wound-field current density $\left(U_{e c}\right)$, to mention a few. The current densities have been varied to ensure that reasonable thermal limits are respected in the final designs, notwithstanding the fact that a failsafe thermal analysis is not an objective in this study. The airgap $(g)$, slot filling ratios of the wound-field DC $\left(k_{e c}\right)$ and armature $\left(k_{s}\right)$ winding slots, as well as rated generator speed $\left(n_{s}\right)$, among others, were kept constant. After defining the design parameters, some additional formulations, necessary for the optimisation process, were implemented to describe realistic boundary conditions, and were kept as similar as possible for both designs. On completion of the optimisation runs, the final designs were collected and compared, as discussed in the following section.

\section{Results and discussions}

The results of the 2D FEA optimisation process are presented in Tables 1 and 2. As indicated, most of the design targets were achieved, especially for D-II. After several optimisation runs, it was proving difficult to realise the efficiency prescription for D-I without exceeding the logical limits of the defined design optimisation space. It is not a surprising occurrence, especially given that the compared evaluated total conductor losses $\left(P_{c u}\right)$ shows that the specific DC conductor losses of D-I is $13.03 \mathrm{~kW}$, while that of DII is a mere $8.64 \mathrm{~kW}$, which is due to the fact that the same slot filling factors $\left(k_{s}\right.$ and $\left.k_{e c}\right)$ were assumed for both designs in 2D FEA. On a closer inspection of the slot designs, such an assumption is naturally more profitable to D-II than to D-I, as shown in Figure 4 . Also, the end-winding effects might have been poorly attributed in D-II, thus resulting in smaller conductor losses.

Table 1: Optimum performance data of $300 \mathrm{~kW}$ wind generators.

\begin{tabular}{lccccccccccccccc}
\hline & $\begin{array}{c}\Delta \tau_{N L} \\
(\%)\end{array}$ & $\begin{array}{c}\Delta \tau_{L} \\
(\%)\end{array}$ & $\begin{array}{c}P_{c u} \\
(\mathrm{~kW})\end{array}$ & $\begin{array}{c}P_{\text {core }} \\
(\mathrm{kW})\end{array}$ & $\begin{array}{c}P F \\
-\end{array}$ & $\begin{array}{c}\eta \\
(\%)\end{array}$ & $\begin{array}{c}T_{r} \\
(\mathrm{kNm})\end{array}$ & $\begin{array}{c}M_{E F S} \\
(\mathrm{~kg})\end{array}$ & $\begin{array}{c}M_{C u} \\
(\mathrm{~kg})\end{array}$ & $\begin{array}{c}M_{F e} \\
(\mathrm{~kg})\end{array}$ & $\begin{array}{c}M_{\text {Tot }} \\
(\mathrm{kg})\end{array}$ & $\begin{array}{c}P_{\text {loss }} / V_{\text {act }} \\
\left(\mathrm{kW} / \mathrm{m}^{3}\right)\end{array}$ & $\begin{array}{c}T_{r} / V_{\text {act }} \\
\left(\mathrm{kNm} / \mathrm{m}^{3}\right)\end{array}$ & $\begin{array}{c}P_{g} \\
(\mathrm{~kW})\end{array}$ \\
\hline D-I & 7.85 & 8.76 & 15.63 & 3.16 & 0.79 & 94.15 & 9.52 & 74.5 & 61.3 & 2183.5 & 2319.3 & 41.38 & 20.86 & 303 \\
D-II & 6.62 & 5.56 & 10.52 & 2.32 & 0.98 & 95.91 & 9.43 & 63.9 & 63.9 & 1744.3 & 1872.1 & 34.29 & 24.79 & 302 \\
PMSG & 5.30 & 25.74 & 13.30 & 2.46 & 0.95 & 95.00 & 67.00 & 114.6 & 282.3 & 967.0 & 1363.9 & 12.11 & 52.50 & 300 \\
\hline
\end{tabular}

Table 2: Optimum design data of $300 \mathrm{~kW}$ wind generators.

\begin{tabular}{|c|c|c|c|c|c|c|c|c|c|c|}
\hline & $\begin{array}{c}J_{S} \\
\left(\mathrm{~A} / \mathrm{mm}^{2}\right)\end{array}$ & $\begin{array}{c}J_{e c} \\
\left(\mathrm{~A} / \mathrm{mm}^{2}\right)\end{array}$ & $\begin{array}{c}n_{s} \\
(r / \mathrm{min})\end{array}$ & $\begin{array}{c}f_{s} \\
(\mathrm{~Hz})\end{array}$ & $\begin{array}{c}l \\
(m)\end{array}$ & $\begin{array}{c}D_{i} \\
(m)\end{array}$ & $\begin{array}{l}D_{o} \\
(m)\end{array}$ & $\begin{array}{c}g \\
(\mathrm{~mm})\end{array}$ & $\begin{array}{c}k_{S} \\
-\end{array}$ & $\begin{array}{c}k_{e c} \\
-\end{array}$ \\
\hline D-I & 3.50 & 7.62 & 300 & 50 & 0.37 & 0.90 & 1.25 & 2 & 0.45 & 0.45 \\
\hline D-II & 3.33 & 7.29 & 300 & 50 & 0.38 & 0.79 & 1.12 & 2 & 0.45 & 0.45 \\
\hline PMSG & 4.58 & - & 50 & 114.6 & 0.26 & 2.43 & 2.5 & - & - & - \\
\hline
\end{tabular}

Another observation in D-I which can be associated with its end-winding evaluation is the recorded lower power factor, which should inherently yield larger leakage reactance compared to D-II. To this end, D-II is seen with a higher power factor, which may imply lower $\mathrm{kVA}$ ratings and inverter losses (costs), and higher generator overload capability. Furthermore, it is observed that D-II yields $18.8 \%$ higher torque density $\left(\mathrm{kNm} / \mathrm{m}^{3}\right)$ and $17.1 \%$ lesser loss per active volume $\left(\mathrm{kW} / \mathrm{m}^{3}\right)$ than D-I.

Meanwhile, note that Tables 1 and 2 also display the performance data of a $300 \mathrm{~kW}$ optimum conventional direct-drive PMSG as evaluated in Potgieter and Kamper. [9]. The performance of the PMSG is carefully contrasted against that of the studied WF-FSM variants designed in the mediumspeed drivetrain. It is observed that the active masses $\left(M_{T o t}\right)$ and torque density $\left(T_{r} / V_{a c t}\right)$ of D-I and D-II is much higher than that of the PMSG. Also, in terms of the $P_{\text {loss }} / V_{\text {act }}$ index, the value for the PMSG is significantly lower than those conceived in the WF-FSM designs. As noted in Potgieter and Kamper. [9], higher loss per active volume as observed in D-I and D-II can result in serious thermal implications, which may need to be carefully addressed.

Table 3: Cost analyses data of $300 \mathrm{~kW}$ wind generators.

\begin{tabular}{lcccc}
\hline & $E F S$ & $C u$ & $F e$ & Total \\
& $(U S D)$ & $(U S D)$ & $(U S D)$ & $(U S D)$ \\
\hline D-I & 834.4 & 686.6 & 4803.7 & 6324.7 \\
D-II & 715.7 & 715.7 & 3837.5 & 5268.9 \\
PMSG & 6876 & 3161.8 & 2127.4 & 12165.2 \\
\hline
\end{tabular}




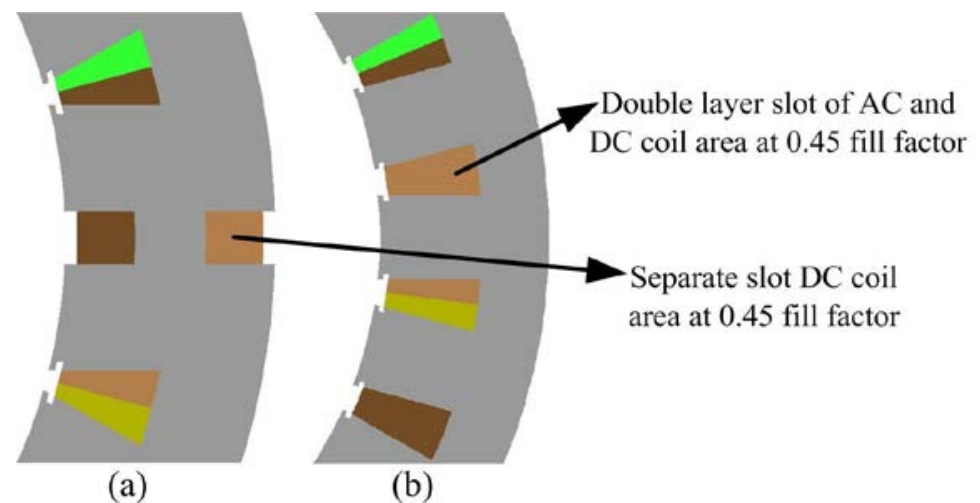

(a)

(b)

Figure 4: Depiction of the slot filling areas in 2D FEA modelling: (a) D-I, and (b) D-II.

The PMSG is designed with rare earth PMs, which should result in a trade-off between the active mass and total costs. Consequently, the cost analyses of the generators are contrasted as shown in Table 3. Clearly, it is observed that although the total active mass of the PMSG is smaller than the WFFSM designs, its total cost is about twice as much. The reverse is the case for the WF-FSM designs, which shows their total masses to be much higher, yet the cost of material is significantly reduced because they are rare earth-free. It should be mentioned that although the comparison between the direct-drive PMSG and the geared WF-FSM designs is not exactly comfortable because of their different drivetrain configurations, yet it gives insight to the fact that the avoidance of rare earth PMs may positively impact on the latter's total cost of materials.

\section{3D FEA validation and experimental pro- totype}

This section presents the 3D FEA validation of the studied WF-FSMs, as well as experimental results achieved from an existing $10 \mathrm{~kW}$ prototype of D-I designed, built and tested as a wind generator in the Electrical Machines Laboratory of Stellenbosch University, South Africa, in 2017, for the first time [15].

\subsection{D FEA Validation}

In Figure 5, the flux density plots of the $300 \mathrm{~kW}$ WFFSMs evaluated in 3D FEA as quarter symmetrical models are presented. The peak density flux in the iron cores reaches $2.3 \mathrm{~T}$ in trace proportions in regions close to the airgap, at rated conditions. Table 4 is then introduced by way of comparing the 2D and 3D FEA evaluations to fully account for the endwinding effects. It is clear enough that the end-winding effects were seriously underestimated in D-II, as witnessed by the significant increase in the total copper losses. This huge drop has to be implicated in the discrepancy observed in the average torque, torque density and efficiency. The highlight of endwinding effects is the reversal of the $P_{\text {loss }} / V_{\text {act }}$ index between the machines, which suggests in reality that it may be worse for D-II than D-I. Also, a huge difference in the torque ripple of D-II is also observed. The trend, which appeared to be steadfast in 2D and $3 \mathrm{D} F E A$, is that the torque density of the D-II is slightly maintained above that of D-I, as well as attainment of unitary power factor in the former.

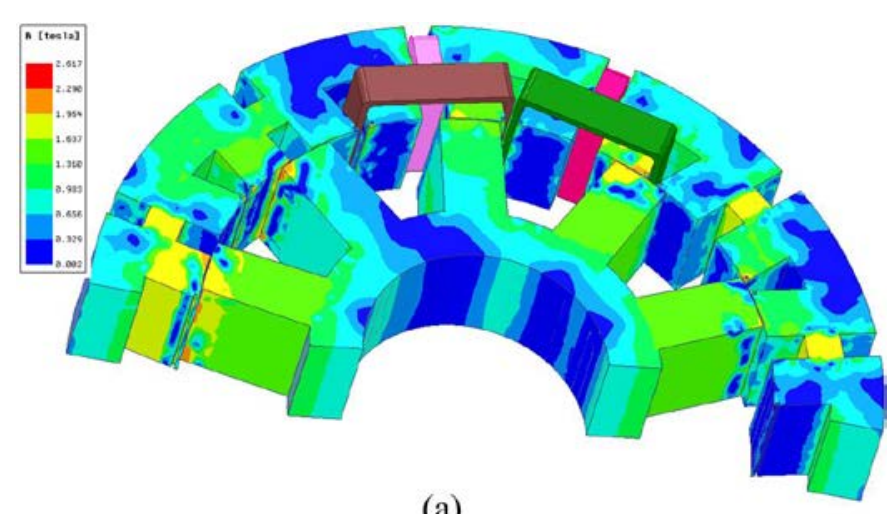

(a)

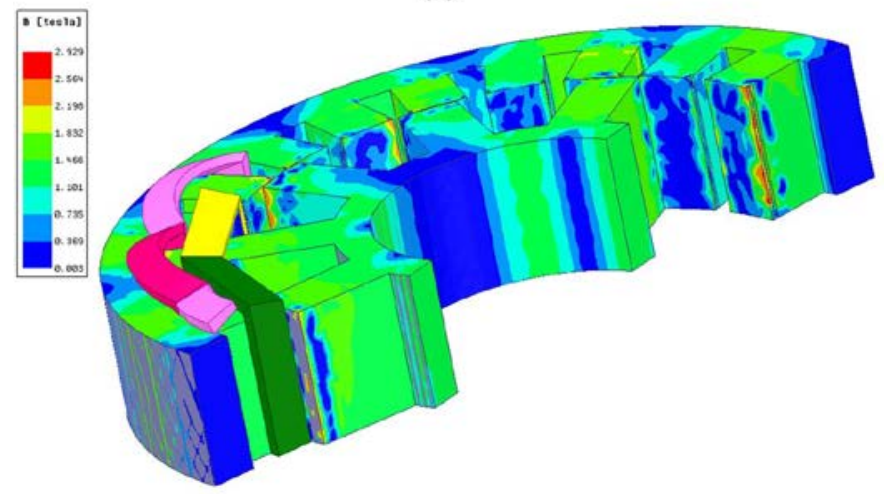

(b)

Figure 5: 3D FEA predicted flux density contour plots of the studied $300 \mathrm{~kW}$ WF-FSMs at rated conditions: (a) D-I, and (b) D-II.

\subsection{Experimental prototype and tests}

The main handout from the experimental demonstration is that the manufacturing is $100 \%$ South African, without the need for importation of any of the resource components used in the fabrication process. In Figure 6, the highlights of the manufacturing process are photographically profiled. The test 
bench is shown in Figure 7, while Figure 8 is used to gauge some of the measured results with those predicted initially at the design stage. At higher loading, it appears the effect of saturation becomes daring, as observed in Figure 8. Other than that, a good agreement is indicated. The level of agreement achieved through the experimental testing of the 10 $\mathrm{kW}$ prototype yields some promise as to the feasibility of furthering the power levels to the proposed $300 \mathrm{~kW}$ wind generator concepts for South Africa's wind power industry, as already being initiated in this study.

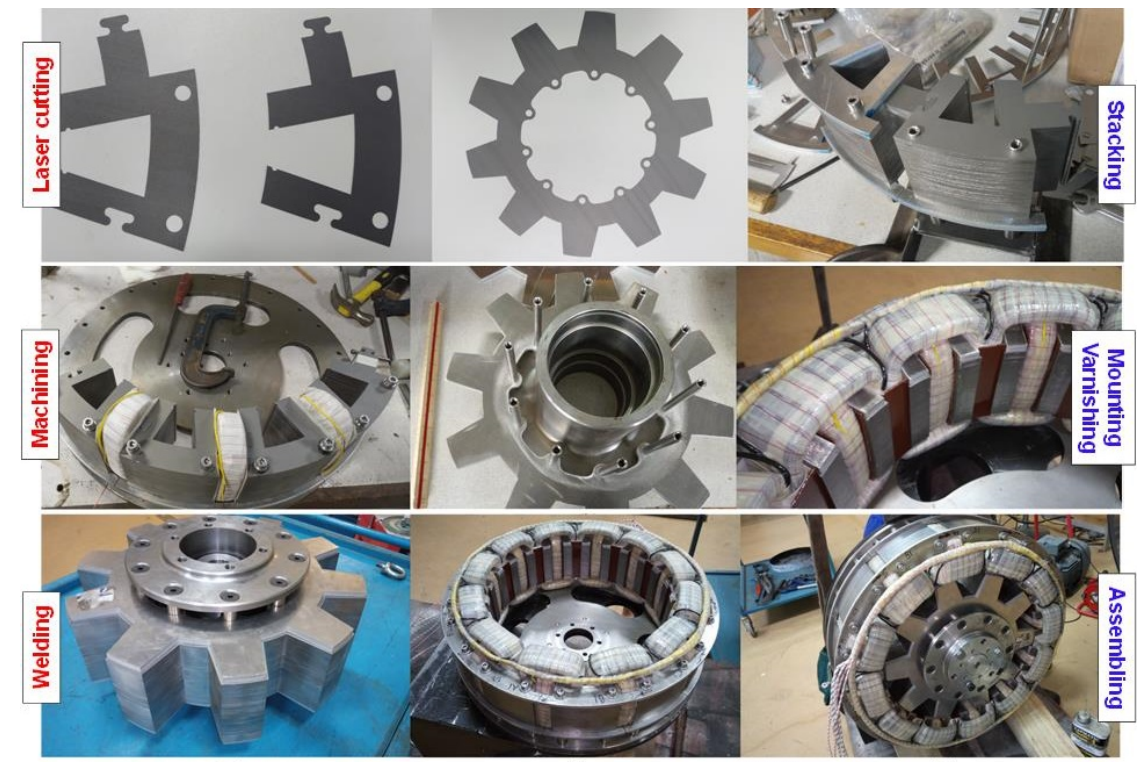

Figure 6: Manufacturing process of a $10 \mathrm{~kW}$ WF-FSM wind generator prototype [15].

Table 4: Comparison between 2D and 3D FEA performance data of $300 \mathrm{~kW}$ wind generators.

\begin{tabular}{cccccccccccccccccc}
\hline & \multicolumn{2}{c}{$\begin{array}{c}\Delta \tau_{L} \\
(\%)\end{array}$} & \multicolumn{2}{c}{$\begin{array}{c}P_{c u} \\
(\mathrm{~kW}\end{array}$} & \multicolumn{2}{c}{$\begin{array}{c}P_{\text {core }} \\
(\mathrm{kW}\end{array}$} & $\begin{array}{c}T_{r} \\
(\mathrm{kNm})\end{array}$ & $\begin{array}{c}\eta \\
(\%)\end{array}$ & $\begin{array}{c}P_{\text {loss }} / V_{\text {act }} \\
\left(\mathrm{kW} / \mathrm{m}^{3}\right)\end{array}$ & \multicolumn{2}{c}{$\begin{array}{c}T_{r} / V_{\text {act }} \\
\left(\mathrm{kNm} / \mathrm{m}^{3}\right)\end{array}$} & $\begin{array}{c}\text { PF } \\
-\end{array}$ \\
\hline & $2 D$ & $3 D$ & $2 D$ & $3 D$ & $2 D$ & $3 D$ & $2 D$ & $3 D$ & $2 D$ & $3 D$ & $2 D$ & $3 D$ & $2 D$ & $3 D$ & $2 D$ & $3 D$ \\
D-I & 8.76 & 8.85 & 15.63 & 15.60 & 3.16 & 3.56 & 9.52 & 9.03 & 94.15 & 93.67 & 41.38 & 42.19 & 20.86 & 19.88 & 0.79 & 0.65 \\
D-II & 5.56 & 17.96 & 10.52 & 16.08 & 2.32 & 2.09 & 9.43 & 8.24 & 95.91 & 93.44 & 34.29 & 48.53 & 24.79 & 22.00 & 0.98 & 1.00 \\
\hline
\end{tabular}

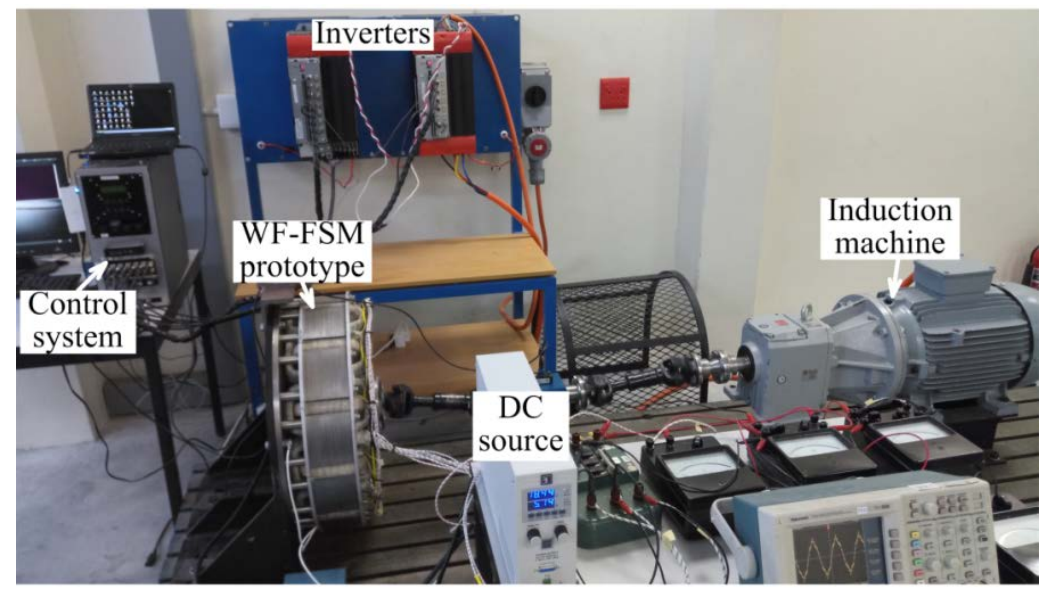

Figure 7: WF-FSM wind generator prototype being tested on laboratory test bench [15]. 


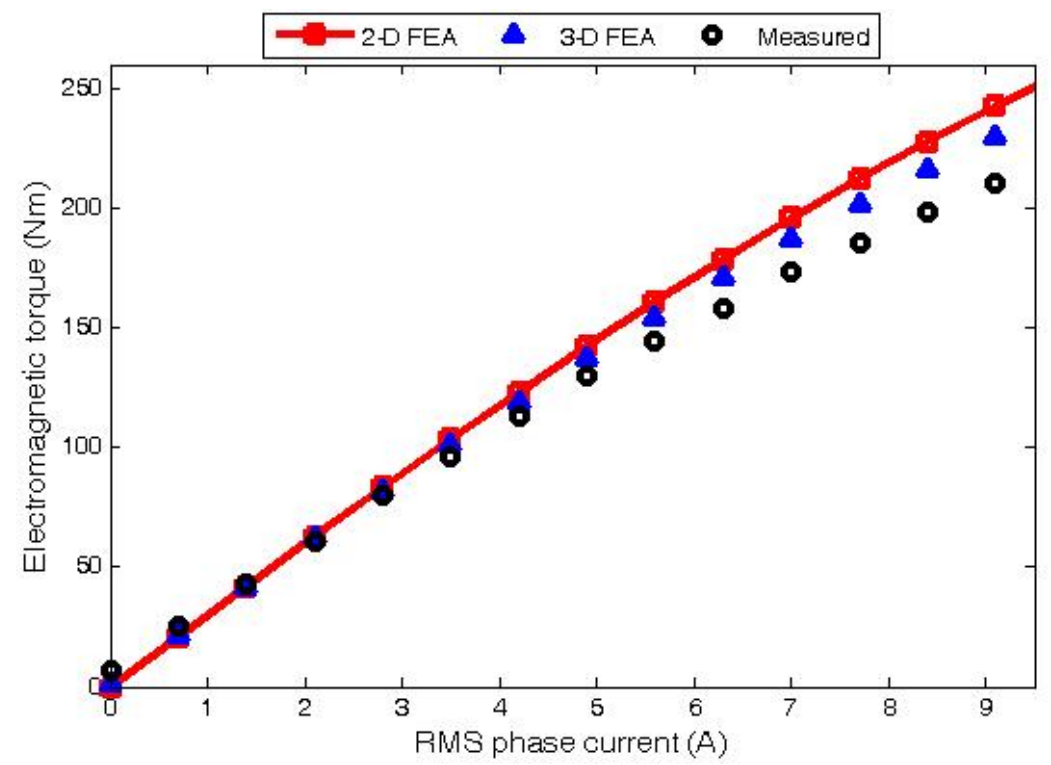

Figure 8: Measured electromagnetic torque under varying load current [11].

\section{Conclusions}

In this paper, an optimal design comparison has been initiated for two wound-field flux switching machine variants, profiled as D-I and D-II, at $300 \mathrm{~kW}$ power ratings, in order to demonstrate the industrial relevance of rare earth-free wind generators to the booming South Africa wind power industry. In 2D finite element analyses (FEA), D-II shows better performance credentials in response to the specified design constraints, but with a significant discrepancy in the specific direct current copper losses observed due to unfair approximations of the slot filling factors. However, after undertaking 3D FEA, the results show that the end-winding effects of D-II have been seriously underrated, of which among other things, the $P_{\text {loss }} / V_{\text {act }}$ index increases by $15 \%$ compared to
D-I. However, the torque density and power factor of D-II remains steadfastly higher than D-I, even in 3D FEA. At the time of writing, a locally manufactured $10 \mathrm{~kW}$ laboratory prototype of D-I existed, ensuring that some experimental validation is provided. In conclusion, the study uncovers some degree of design feasibility with perceived economic benefits of the proposed wind generators for South Africa's wind power industry.

\section{Author roles}

U.B. Akuru designed the research, did the analysis and the technical report.

M.J. Kamper provided guidelines on the research techniques and inputs on the write-up.

\section{References}

[1] REN21, Renewables 2018 global status report (Paris: REN21 Secretariat), 2018. Available: www.ren21.net/gsr.

[2] Draft IRP-2018: Integrated Resource Plan - Department of Energy, Republic of South Africa, August 2018. Accessed online on 2018/10/16 at: http://www.energy.gov.za/IRP/irp-update-draft-report2018/IRP-Update-2018Draft-for-Comments.pdf

[3] Goudarzi, N. and Zhu, W. D. 2013. A review on the development of wind turbine generators across the world. International Journal of Dynamics and Control 2013(1): 192-202. https://doi.org/10.1007/s40435-0130016-y

[4] Keysan, O. 2017. Future electrical generator technologies for offshore wind turbines, Wind Power, E\&T IET Services, Aug 16, 2017. Accessed online on 2018/07/12 at: https://energyhub.theiet.org/users/60216-ozankeysan/posts/19442-future-electrical-generator-technologies-for-offshore-wind-turbines

[5] Pavel, C. C., Lacal-Arántegui, R., Marmier, A., Schüler, D., Tzimas, E., Buchert, M., Jenseit, W. and Blagoeva, D. 2017. Substitution strategies for reducing the use of rare earths in wind turbines. Resources Policy 52: 349-357. https://doi.org/10.1016/j.resourpol.2017.04.010

[6] VENPOWER: http://www.venpower.de/index.html

[7] Rauch, S. E. and Johnson, L. J. 1955. Design principles of flux-switching alternators. AIEE Transactions 74(3): 1261-1268. https://doi.org/10.1109/AIEEPAS.1955.4499226

[8] Cheng, M., Hua, W., Zhang, J. and Zhao, W. 2011. Overview of stator-permanent magnet brushless machines. IEEE Transactions on Industrial Electronics 58(11): 5087-5101. https://doi.org/10.1109/TIE.2011.2123853 
[9] Potgieter, J. H. J. and Kamper, M. J. 2016. Double PM-rotor, toothed, toroidal-winding wind generator: A comparison with conventional winding direct-drive $\mathrm{PM}$ wind generators over a wide power range. IEEE Transactions on Industry Applications 52(4): 2881-2891, 2016. https://doi.org/10.1109/TIA.2016.2536580

[10] Kamper, M. J. 2009. Development and test results of South Africa's first $300 \mathrm{~kW}$ permanent magnet direct drive grid connected wind generator system (discussion paper). 18th Southern African Universities Power Engineering Conference (SAUPEC), Stellenbosch, South Africa, 2009.

[11] Akuru, U. B. and Kamper, M. J. 2018. Intriguing behavioural characteristics of rare-earth-free flux switching wind generators at small- and large-scale power levels. IEEE Transactions on Industry Applications 54(6): 5772-5782. https://doi.org/10.1109/TIA.2018.2848979

[12] SEMFEM documentation: www0.sun.ac.za/semfem/index.html

[13] VisualDOC user's manual: Version 8.0, Vanderplaats Research \& Development, Inc., Colorado Springs, CO, February 2017.

[14] Vanderplaats, G. N. 2007. Multidiscipline design optimisation. Colorado Springs, USA: Vanderplaats Research \& Development, Inc.

[15] Akuru, U. B. and Kamper, M. J. 2018. Novel experimentation of a $10 \mathrm{~kW}$ geared medium-speed wound-field flux switching wind generator drive. 2018 IEEE Energy Conversion Congress and Exposition, Portland, Oregon, September 23-27, 2018: 6492-6498. https://doi.org/10.1109/ECCE.2018.8557762. 